\title{
The Influence of Bonding System Type on Strength Properties of Reconstructed Teeth with 1st Class Cavities According to Black's Classification
}

\author{
Grzegorz Milewski ${ }^{1, a}$, Agata Borowiec ${ }^{1, b}$ \\ ${ }^{1}$ Cracow University of Technology, Institute of Applied Mechanics, Warszawska 24, 31-155 \\ Cracow, Poland \\ amilewski@mech.pk.edu.pl, bborowiec.agata@gmail.com
}

\begin{abstract}
Keywords: Dental Bonding System, First Class Tooth Cavity Reconstruction, Strength Properties
\end{abstract}

\begin{abstract}
The paper presents the results of strength tests of lateral teeth with $1^{\text {st }}$ class cavities reconstructed with various bonding systems, i.e. self- and selective bonding systems as well as for filling with additional etching with orthophosphoric acid. In all cases a nano-hybrid, lightcured composite material was used in a layered (sandwich) cavity filling processing. The strength tests were done with the use of Instron 4465 strength machine for loadings simulating the features of proper lateral teeth occlusion. The analyses of the results were done with respect to the following quantities: ultimate force, total displacement and work to fracture. The results were compared with reference 'healthy' lateral teeth group. Analysis of the results proves that the type of bonding system has a significant effect on the values of individual strength parameters of the tested tooth samples. From the mechanical point of view the best results were obtained for self- and selective bonding system when comparing with additional etching procedure. However, regardless on the type of bonding system, the mechanical properties of teeth subjected to dental treatment are significantly lower than those for the 'healthy' teeth.
\end{abstract}

\section{Introduction}

Adhesion is the basic physical phenomenon that determines the success of composite crown fillings in contemporary conservative dentistry [1]. Phenomenon of adhesion consisting in the combination of surface-contact layers of two bodies in a solid or liquid phase combines with the mechanical, polarizing, diffusive and adsorptive theories of the mutual interaction of two bodies. Close contact of the contacting bodies is necessary for the formation of the adhesion phenomenon - the distance between the bodies should be smaller than 1 - $2 \AA(10-20 \mathrm{~nm})$ Fig. 1a. In practice, achieving such a low distance between solid bodies is difficult to achieve. Therefore, in order to obtain adhesion forces, a liquid photo polymerized material is used to wet the substrate. This material, called a bonding system, acts as an intermediate adhesive layer and provides better connection with dental fillings. The bonding systems have been developed over years. The differences between all generations of bonding systems are significant. Development of materials and techniques have changed the bonding systems themselves as well as their adhesive properties and strength [2]. In general, adhesion is determined by the value of the unitary adhesion forces or the work necessary to separate the adherent bodies, however various physical quantities are used to quantify that phenomenon between hard tissues of tooth (i.e. dentine and enamel) and dental composite filling material. The aim of the work was to determine the strength properties of reconstructed lateral tooth crowns with the use of various bonding systems. 


\section{Materials and Methods}

Black's scale is the most often used classification in contemporary conservative dentistry. It is based on the tooth type and the cavity location or tooth surfaces involved. Black's scale consists of (I - VI) classes [1]. The first class cavities are located in pits or fissures of crowns, mainly in the occlusal surfaces of molars and premolars as well as in the lingual surfaces of upper incisors, and occasionally in the lingual surfaces of upper molars - Fig. 1 b.

a.

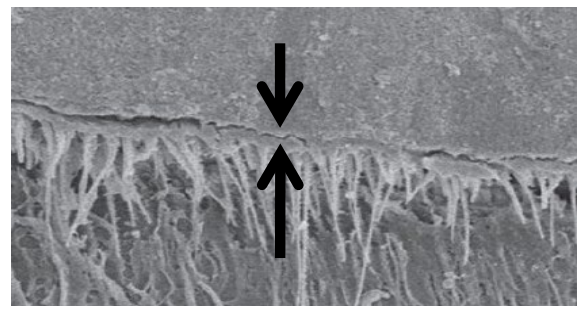

b.

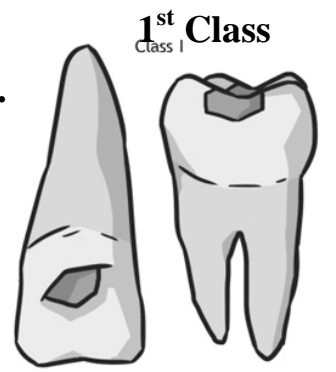

c.

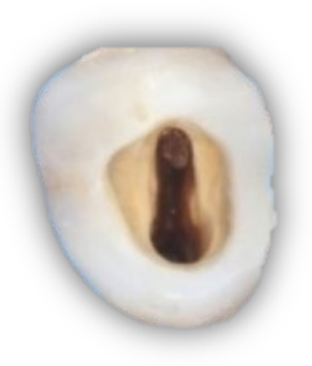

Fig. 1. SEM image of adhesive layer between dentine and composite filling material (a); scheme of $1^{\text {st }}$ class cavity according to Black's classification (b) and tooth crown cavity preparation after endodontic treatment ready for final reconstruction (c)

The tests were done for 15 samples - lateral teeth after extraction for orthodontic reasons. All teeth were prepared for the $1^{\text {st }}$ class cavities according to Black's classification. The teeth were randomly divided into three reconstructive groups (5 samples in each group): I - teeth reconstructed with self-bonding system that does not require additional etching procedure, II reconstruction with additional etching with orthophosphoric acid, III - teeth reconstructed with the use of a selective-etching bonding system - Fig. 2. All teeth-samples were prepared under a supervision of a dentist and were done with standard dental treatment procedures. A nanohybrid, light-cured composite material was used in the reconstruction of all teeth crowns, which were worked out with layered (sandwich) cavity filling processing [1, 3].

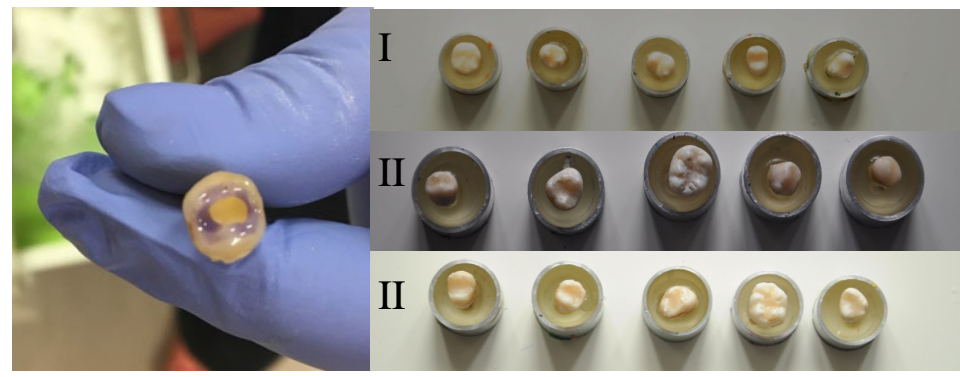

I - teeth reconstructed with a bonding system that does not require additional etching with orthophosphoric acid II - reconstruction with additional etching

III - teeth reconstructed with the use of a selective bonding system

Fig. 2. Set of tooth samples with crowns reconstructed with the use of various bonding systems

The tests were done with the use of Instron 4465 strength machine for loadings simulating the features of proper teeth occlusion. As there is no standardized method of strength tests of tooth crowns a compression test with a steel ball that is used instead of an opposing tooth was applied. The method was proposed and developed in $[4,5,6]$. The following quantities were registered and analyzed: ultimate force $F_{c}$, work to fracture $W_{c}$, total displacement $u_{c}$. The results of the 
strength tests in all groups were compared with the respective results for reference 'healthy' lateral teeth (group 0) strength tests which were done in the previous author's work [4].

\section{Results and Discussion}

The results of strength tests carried out for application of three different bonding systems for $1^{\text {st }}$ class cavities in lateral teeth treatment are presented in Table 1. The data are given for mean values and standard deviations (SD) for each respective teeth groups.

Table 1. Set of registered and analyzed values of ultimate force $F_{c}$, total displacement $u_{c}$ and work to fracture $W_{c}$ for lateral teeth groups reconstructed with various bonding systems

\begin{tabular}{|c|c|c|c|c|c|c|}
\hline \multirow{2}{*}{$\begin{array}{c}\text { Teeth } \\
\text { group }\end{array}$} & \multicolumn{2}{|c|}{ Ultimate force [kN] } & \multicolumn{2}{c|}{ Displacement [mm] } & \multicolumn{2}{c|}{ Work to fracture [J] } \\
\cline { 2 - 7 } & mean & SD & mean & SD & mean & SD \\
\hline 0 & 1.850 & 0.1996 & 2.162 & 0.1032 & 1.530 & 0.0873 \\
\hline I & 1.005 & 0.2849 & 1.433 & 0.7116 & 0.599 & 0.3969 \\
\hline II & 0.563 & 0.1783 & 1.714 & 0.7940 & 0.291 & 0.0854 \\
\hline III & 0.947 & 0.1486 & 1.614 & 0.7399 & 0.574 & 0.1910 \\
\hline
\end{tabular}

The analysis consisted of comparison of all specimens data in each group and also of comparison of mean values of each parameter in the selected groups. All used types of bonding systems were compared with each other. The analyses were done with regards to the respective values obtained for the 'healthy' teeth. The obtained results allow to conclude that all cavities reconstructions, regardless the bonding system type, decrease the mechanical properties of teeth. The smallest differences between the destructive forces $\left(F_{c}\right)$ occur for self-bonding system (group I). The difference between these values is around $45 \%$. Similar values were obtained for the third research group, here the difference is about $48 \%$, while the difference between the respective values of ultimate forces for the system requiring additional etching (group II) is almost 70\%. For the work to fracture $\left(\mathrm{W}_{\mathrm{c}}\right)$ the relevant ratios are even higher and reaches $61 \%, 64 \%$ and $81 \%$ respectively, what stands for the essential reduction of the ability to energy dissipation for lateral teeth crowns subjected to dental treatment. This regularity is illustrated by a histogram presented in Fig. 3.

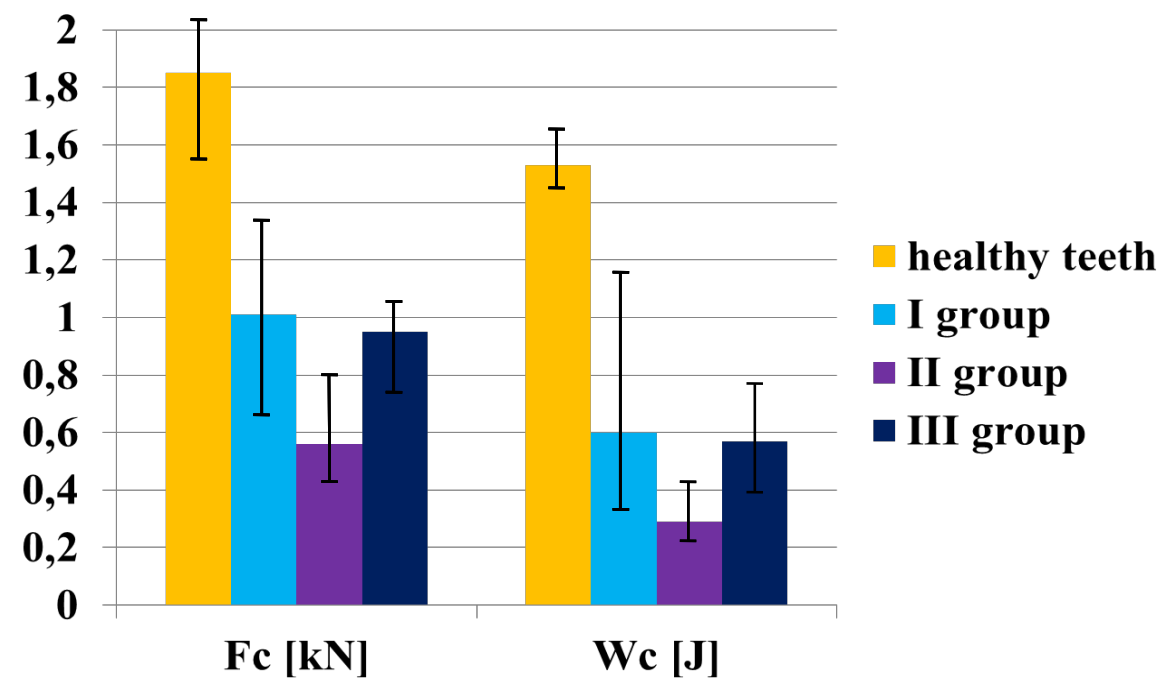

Fig. 3. Comparison of the mean values of ultimate force $F_{c}$ and work to fracture $W_{c}$ for lateral teeth crowns reconstructed with the use of various bonding system 


\section{Summary}

Analysis of the results proves that the type of bonding system has a significant effect on the values of individual strength parameters of the tested tooth samples. The best results were obtained for self- and selective-etching bonding system. However, regardless the type of bonding system, the mechanical properties of teeth subjected to dental treatment are significantly lower than those for the 'healthy' teeth. Samples damage analysis shows that in general there was no delamination of the composite material what proves the good strength properties of all bonding systems used in the research. The study shows that there is a need for further development of bonding systems and dental fillings in order to increase their mechanical properties.

\section{References}

[1] R. Ireland (Ed.), A Dictionary of Dentistry, Oxford University Press, Oxford, New York, 2010

[2] R. L. Sakaguchi, J. M. Powers (Eds.), Craig's Restorative Dental Materials, 13th Edition, Elsevier/ Mosby, Philadelphia, 2012

[3] G. Milewski, T. Majewski, Influence of the method of polymerization of composite material for teeth crown fillings on its strength properties, Solid State Phenomena (2016), 240, pp. 168173. https://doi.org/10.4028/www.scientific.net/ssp.240.168

[4] G. Milewski, Numerical and experimental analysis of effort of human tooth hard tissues in terms of proper occlusal loadings, Acta of Bioengineering and Biomechanics (2006), 7 (1), pp. 47-58

[5] R. Sorrentino, Z. Salameh, F. Zarone, F.R. Tay, M. Ferrari, Effect of post-retained composite restoration of MOD preparations on the fracture resistance of endodontically treated teeth, Journal of Adhesive Dentistry (2007), 9 (1), pp. 49-56

[6] G. Milewski, A. Hille, Experimental strength analysis of orthodontic extrusion of human anterior teeth, Acta of Bioengineering and Biomechanics (2012), 14(1), pp. 15-21 